\title{
Uso de psicoestimulante por acadêmicos de medicina em instituição de ensino superior na Amazônia Ocidental
}

\author{
Use of psychostimulant by medicine academics in higher education institution in Western \\ Amazon
}
Uso del psicostimulante por los académicos de la medicina em la institución de educación superior em la Amazônia Occidental

Ingrid Zandoná1*, Anitha de Cássia Ribeiro da Silva ${ }^{1}$, Mariana Moraes Cavatti ${ }^{1}$, Jorge Victor Moreira Aguiar $^{1}$, Kemilly Teixeira de Andrade ${ }^{1}$, Camylla Felix Soares ${ }^{1}$, Mayara Gonçalves Tavares ${ }^{1}$, Camila Maciel de Sousa ${ }^{1}$.

\section{RESUMO}

Objetivo: Avaliar o uso de psicoestimulantes entre acadêmicos de medicina em uma instituição de ensino superior na Amazônia Ocidental. Métodos: Trata-se de um estudo de caráter descritivo, exploratório, transversal e de abordagem quantitativa, realizado com 265 estudantes do primeiro ao sexto ano, maiores de 18 anos, por meio do preenchimento de questionários abrangendo questões relacionadas ao uso de psicoestimulantes. Os dados obtidos foram analisados com o auxílio do programa Microsoft Office Excel. Resultados: Segundo a amostra, $66,4 \%$ eram do sexo feminino e $33,5 \%$ do masculino. A idade média foi de 19 a 23 anos. No que tange ao uso de psicoativo, 65,2\% afirmaram fazer uso de algum psicoestimulante, sendo que, $45,6 \%$ relataram regular ingestão e 19,6\% informaram utilizar apenas no período avaliativo. Entre os principais motivos citados para o consumo foram: $57,7 \%$ devido a rotina e $24,1 \%$ pelo aumento da capacidade cognitiva. Mais de $57 \%$ consideram o uso de psicoestimulantes com melhora na rotina acadêmica, além de ajudar no aumento da capacidade cognitiva e compensar a privação do sono. Conclusão: Concluise que o uso de psicoativos se apresenta frequente na rotina dos acadêmicos de medicina, principalmente no período de provas.

Palavras-Chave: Substâncias para melhoria do desempenho, Estudantes, Medicina, Dependência.

\section{ABSTRACT}

Objective: To evaluate the use of psychostimulants among medical students in a higher education institution in the Western Amazon. Methods: This is a descriptive, exploratory, cross-sectional study with a quantitative approach, conducted with 265 students from the first to the sixth grade, over 18 years old, by completing questionnaires covering questions related to the use of psychostimulants. The data obtained were analyzed with the aid of the Microsoft Office Excel program. Results: According to the sample, $66.4 \%$ were female and $33.5 \%$ male. The average age was 19 to 23 years. Regarding the use of psychoactive, $65.2 \%$ said they use some psychostimulant, and $45.6 \%$ reported regular ingestion and $19.6 \%$ reported use only in the evaluation period. Among the main reasons cited for consumption were: $57.7 \%$ due to routine and $24.1 \%$ due to increased cognitive ability. More than $57 \%$ consider the use of psychostimulants to improve their academic routine, as well as helping to increase cognitive capacity and compensate for sleep deprivation. Conclusion: It is concluded that the use of psychoactive drugs is frequent in the routine of medical students, mainly during the testing period.

Key words: Substances for performance improvement, Students, Medicine, Dependence.

${ }^{1}$ Centro Universitário São Lucas (UNISL), Porto Velho - RO. *E-mail: ingrid_zandona@hotmail.com Iniciação Cientifica dentro do Programa PIBIC/CNPq, n 128503/2018-1 


\section{RESUMEN}

Objetivo: Evaluar el uso de psicoestimulantes entre estudiantes de medicina en una institución de educación superior em la Amazonía occidental. Métodos: Este es um estudio descriptivo, exploratorio, transversal com un enfoque cuantitativo, realizado con 265 estudiantes del primer al sexto grado, mayores de 18 años, completando cuestionarios que cubren preguntas relacionadas con el uso de psicoestimulantes. Los datos obtenidos se analizaron com la ayuda del programa Microsoft Office Excel. Resultados: Según la muestra, $66.4 \%$ eran mujeres y $33.5 \%$ hombres. La edad promedio fue de 19 a 23 años. Con respecto al uso de drogas psicoactivas, el $65.2 \%$ informó haber usado algún psicoestimulante, y el $45.6 \%$ informó haber ingerido regularmente y el $19.6 \%$ informó haberlo usado solo durante el período de evaluación. Entre las principales razones citadas para el consumo se encuentran: $57.7 \%$ debido a la rutina y $24.1 \%$ debido a una mayor capacidad cognitiva. Más del $57 \%$ considera el uso de psicoestimulantes para mejorar su rutina académica, además de ayudar a aumentar la capacidad cognitiva y compensar la falta de sueño. Conclusión: Se concluye que el uso de drogas psicoactivas es frecuente em la rutina de los estudiantes de medicina, principalmente durante el período de prueba.

Palabras clave: Sustancias para la mejora del rendimiento, Estudiantes, Medicina, Dependencia.

\section{INTRODUÇÃO}

O uso de psicoestimulantes é uma pertinente realidade na contemporaneidade. Tal cenário é influenciado devido à necessidade de corresponder às exigências diárias que muitas vezes ultrapassam os limites corpóreos e psicológicos dos indivíduos, o que incita a ingestão desses compostos. Essas substâncias atuam no sistema nervoso central e são divididos em categorias: como os sintéticos e orgânicos, sendo o primeiro grupo representado pelas anfetaminas e taurinas e o segundo pela cafeína e guaranina (SILVEIRA VI, et al., 2015).

Esses compostos abrangem um grupo de drogas que têm ações como: aumento da atividade motora e a redução da necessidade de sono, sendo assim, diminuem a fadiga, induzem a euforia e apresentam efeitos simpaticomiméticos (LAGE DC, et al., 2015). Devido proporcionar tais efeitos, passam a serem usados por estudantes, uma vez que o âmbito acadêmico propicia uma intensa rotina que culmina na utilização dessas substâncias para o aumento do rendimento. Essa realidade presente no cotidiano de jovens do Brasil e do mundo está cada vez mais relacionada ao uso excessivo de álcool e outras drogas lícitas e ilícitas, sendo considerado um problema de saúde pública, devido aos seus determinantes e condicionantes culturais, éticos e econômicos (DÁZIO EMR, et al., 2016).

O intuito da administração dessas substâncias, sendo a cafeína uma das mais utilizadas, relaciona-se com a tentativa de burlar a fadiga das várias horas de estudos e corresponder as cobranças semestrais. Além disso, os principais motivos para o uso também se referem à compensação da privação do sono e ao aumento da concentração (MORGAN HL, et al., 2017). Além desses efeitos, a cafeína atua na capacidade de formular pensamentos, redução do cansaço, melhora na capacidade de executar atividades motoras e possui efeito na concentração plasmática de dopamina, um neurotransmissor relacionado com a sensação de prazer, o que demonstra um dos motivos para o desenvolvimento da dependência com o seu uso (ALMEIDA DVPD, et al., 2013).

Uma substância relevante é o metilfenidato, conhecido popularmente como ritalina, que devido a sua ajuda na melhora da concentração, passou a ser utilizado por quem busca aumentar o rendimento acadêmico e a concentração para realizar tarefas de forma mais produtiva. Entretanto, quando utilizado de forma inadequada e sem acompanhamento médico pode causar efeitos adversos como: nervosismo, dificuldade para conciliar o sono, diminuição do apetite, cefaleia, palpitação, boca seca e alterações cutâneas (AFFONSO RS, et al., 2016).

Vale ressaltar, que a utilização dessas substâncias está relacionada a problemas emocionais como ansiedade, depressão, medo, angústia e altos níveis de estresse. Atrelado a isto há o uso de drogas como 
maconha, cocaína e álcool, que podem acarretar dependência química, alterações psicológicas e comportamentais (MENDES SV, et al., 2015). Esses fatores podem refletir na redução da expectativa de vida, visto que, haverá maior suscetibilidade a acidentes automobilísticos, relações sexuais desprotegidas e consequente transmissão de DST's, distúrbios do sono, ansiedade, mudanças no hábito alimentar, e estresse, além de causar prejuízos acadêmicos. Desse modo, é necessário que as universidades analisem os fatores que influenciam os alunos a fazerem o uso indiscriminado dessas substâncias (DÁZIO EMR, et al., 2016).

Com base nos dados expostos, observa-se que a busca por meios de otimização do rendimento é uma realidade crescente na comunidade acadêmica e no anseio de bons resultados para poder galgar boa colocação no mercado de trabalho. Dessa forma, presente trabalho buscou avaliar o uso de psicoestimulantes pelos acadêmicos de medicina.

\section{MÉTODOS}

Este estudo é de caráter descritivo exploratório, realizado com estudantes que cursam do primeiro ao sexto ano do curso de medicina em uma Instituição de Ensino Superior Privada da capital do Estado de Rondônia. O Curso de Medicina é composto por 12 turmas (semestres). Sendo que, as turmas do primeiro ao quarto período são compostas por cerca de 100 alunos cada. As turmas do quinto ao décimo segundo períodos com cerca de 50 alunos cada. Assim, temos aproximadamente 800 alunos.

É importante ressaltar que o estudo foi realizado ao longo do segundo semestre de 2018, por isso é impossível determinar o número de alunos, pois há variação ao longo do semestre letivo. Sendo assim, foi utilizado 800 como número para fins de cálculo amostral. Para o cálculo amostral foi definido um nível de confiança de $95 \%$ e uma margem de erro de 0,05. Com isso a amostra mínima estipulada foi de 250 indivíduos. Os indivíduos foram selecionados de forma randomizada simples. Além disso, ao final do trabalho da coleta de dados foram obtidos 265 questionários completos.

Este projeto está sendo realizado após aprovação do Comitê de Ética em Pesquisa em uma Instituição de Ensino Superior Privada no município de Porto Velho, RO, conforme a Resolução 466/12, Comissão Nacional de Saúde/ Ministério da Saúde, de 13/06/13. Comitê de Ética em Pesquisa (CAAE 95118718.0.0000.0013). Os estudantes foram convidados a participarem da pesquisa, onde foi explicado sobre o projeto e após a assinatura do Termo de Consentimento Livre e Esclarecido (TCLE) proposto aos acadêmicos responderem um questionário. O questionário é composto por 12 questões divididas em: gênero, idade, ano do curso de medicina, frequência do uso de café, se é fumante, uso de psicoativos, horas e qualidade do sono, se há uso de medicações para dormir, quais psicoativos foram usados de forma indiscriminada, em qual momento do curso aconteceu o uso, quais os efeitos percebidos após o uso deles e os motivos que levaram o estudante a fazer o uso.

Além disso, para evitar omissão de informações e constrangimento no momento da entrega do questionário, foi proposto aos participantes que ao terminar o preenchimento do questionário, seria necessário inserir o mesmo em uma caixa, que seria recolhida ao final da aula, evitando assim a identificação do voluntário no momento da entrega. Após os dados serem passados para planilhas em programas específicos no computador, os questionários foram destruídos em máquina picotadora de papel.

Entre os critérios de inclusão, foram inclusos os estudantes maiores de 18 anos que cursam do primeiro ao sexto ano do Curso de Medicina em uma Instituição de Ensino Superior Privada na capital do Estado de Rondônia, que concordaram em participar da pesquisa e, assinaram o TCLE fornecido no ato do acordo. Sendo assim, ocorreu a exclusão dos estudantes menores de 18 anos, que não cursavam Medicina, que não concordaram em participar e/ou não assinaram o TCLE. Ao participar do estudo o estudante se expõe a risco mínimo. $\mathrm{O}$ acadêmico pode se sentir constrangido em respondê-lo. Assim, ao final da pesquisa o grupo se responsabilizará por palestras entre outros meios de comunicação para sensibilizar os acadêmicos sobre a necessidade do uso racional destes fármacos, objetivando seguir seus protocolos de receita. O processamento dos dados foi realizado de forma computadorizada, com formatação de tabelas, comparação entre as variáveis, com o auxílio do programa Microsoft Office Excel 2007. 


\section{RESULTADOS}

Foram aplicados 265 questionários, de um total estimado de 800 alunos, distribuídos uniformemente entre os acadêmicos do primeiro ao sexto ano, obtendo uma taxa de resposta de $97,4 \%$, não ocorrendo nenhuma recusa. No que tange ao sexo mais prevalente, o feminino apresentou maior participação, com $66,4 \%$ dos questionários respondidos. Ao avaliar a idade, a média apresentada foi de 23,3 anos e a faixa etária mais prevalente foi a de 18 a 23 anos.

Entre as variáveis estudadas, foi obtido que, $90,5 \%$ dos entrevistados $(n=240)$ tomam café diariamente. $45,6 \%(n=121)$ declararam fazer o uso de psicoativos, e $19,6 \%(n=52)$ disseram fazer o uso apenas no período de provas. Já, a média de horas de sono diárias entre os estudantes foi de 6,4 horas, sendo que $17,3 \%(n=46)$ dos entrevistados dormiam menos de seis horas por dia e 18,4\% $(n=49)$ utilizavam remédios para dormir. Dos entrevistados, $49,8 \%(n=132)$ avaliaram sua qualidade de sono como ruim (Tabela 1).

Tabela 1 - Questionário sobre Hábitos diários.

\begin{tabular}{|c|c|c|}
\hline Variável & $\mathbf{N}$ & $\%$ \\
\hline \multicolumn{3}{|l|}{ Sexo $(n=265)$} \\
\hline Feminino & 176 & 66,4 \\
\hline Masculino & 89 & 33,5 \\
\hline \multicolumn{3}{|l|}{ Idade (anos) } \\
\hline 18 a 23 & 175 & 66 \\
\hline 24 a 29 & 70 & 26,4 \\
\hline 30 a 50 & 20 & 7,5 \\
\hline \multicolumn{3}{|l|}{ Ano do curso } \\
\hline 1으 & 64 & 24,1 \\
\hline 2 & 66 & 24,9 \\
\hline 30 & 34 & 12,8 \\
\hline 4으 & 34 & 12,8 \\
\hline 5은 & 34 & 12,8 \\
\hline 60 & 33 & 12,4 \\
\hline \multicolumn{3}{|l|}{ Toma café diariamente? } \\
\hline Sim & 240 & 90,5 \\
\hline Não & 25 & 9,4 \\
\hline \multicolumn{3}{|l|}{ Fuma ou já Fumou? } \\
\hline Sim & 30 & 11,3 \\
\hline Não & 235 & 88,6 \\
\hline \multicolumn{3}{|l|}{ Já utilizou algum psicoativo? } \\
\hline Sim & 121 & 45,6 \\
\hline Não & 92 & 34,7 \\
\hline Apenas em período de provas & 52 & 19,6 \\
\hline \multicolumn{3}{|l|}{ Sono (horas por dia) } \\
\hline$<6$ & 46 & 17,3 \\
\hline$\geq 6$ & 219 & 82,6 \\
\hline \multicolumn{3}{|l|}{ Qualidade do sono } \\
\hline Boa & 94 & 35,4 \\
\hline Regular & 39 & 14,7 \\
\hline Ruim & 132 & 49,8 \\
\hline \multicolumn{3}{|l|}{ Usa remédio para dormir? } \\
\hline Sim & 49 & 18,4 \\
\hline Não & 216 & 81,5 \\
\hline
\end{tabular}

Fonte: Zandoná I, et al., 2020. 
A partir da análise da utilização de psicoativos dividida por ano de curso, de um total de 64 entrevistados do primeiro ano, cerca de $62,5 \%(n=40)$ confirmaram a utilização de algum psicoativo, desses, $80 \%(n=32)$ afirmaram que sim, por poucas vezes e, $20 \%(n=8)$ afirmaram que sim, apenas no período de provas.

Entre os 66 entrevistados do segundo ano do curso, a confirmação foi de aproximadamente $71,2 \%$ ( $n=$ 47), sendo que $72,3 \%(n=34)$ afirmaram que sim, por poucas vezes e, $27,7 \%(n=13)$ afirmaram que sim, apenas no período de provas. Dos 34 entrevistados do terceiro ano do curso, cerca de $88,2 \%(n=30)$ confirmaram a utilização de algum psicoativo, desses, $70 \%(n=21)$ afirmaram que sim, por poucas vezes e o restante apenas no período de provas.

A partir dos 34 entrevistados do quarto ano do curso, aproximadamente $82,3 \%(n=28)$ confirmaram a utilização de algum psicoativo, e desses, $50 \%(n=14)$ afirmaram que sim, por poucas vezes e a outra metade apenas no período de provas.

Do total de 34 entrevistados do quinto ano do curso, cerca de $32,3 \%(n=11)$ confirmaram a utilização de algum psicoativo, sendo que $81,8 \%(n=9)$ afirmaram que sim, por poucas vezes e, $18,2 \%(n=2)$ afirmaram que sim, apenas no período de provas. Por fim, dos 33 entrevistados do sexto ano do curso, $51,5 \%(n=17)$ confirmaram a utilização de algum psicoativo, e desses, $64,7 \%(n=11)$ afirmaram que sim, por poucas vezes e, $35,3 \%(n=6)$ afirmaram que sim, apenas no período de provas (Tabela 2$)$.

Tabela 2 - Variáveis associadas ao uso de substâncias psicoestimulantes entre os anos do curso de medicina.

\begin{tabular}{cc}
\hline Ano de curso & $\begin{array}{c}\text { \% de alunos que fizeram uso de } \\
\text { psicoestimulantes }\end{array}$ \\
\hline $1 \underline{0}$ & $62,5 \%$ \\
\hline $2 \underline{0}$ & $71,2 \%$ \\
\hline $3 \underline{0}$ & $88,2 \%$ \\
\hline $4 \underline{0}$ & $82,3 \%$ \\
\hline $5 \underline{0}$ & $32,3 \%$ \\
\hline $6 \underline{0}$ & $51,5 \%$
\end{tabular}

Fonte: Zandoná I, et al., 2020.

Em relação à época e o tipo de psicoestimulantes utilizados, o levantamento de dados demonstrou que antes de iniciar a graduação o uso de cafeína foi prevalente entre 191 participantes, sendo representado por $72 \%$, seguido pelo uso de energético correspondendo a $47 \%(n=125)$ do total. Quanto ao uso de ritalina e maconha ambos corresponderam a $14 \%(n=38)$ do espaço amostral, os menores índices de ingestão correspondeu ao ecstasy representando $3 \%(n=9)$.

Ao analisar o uso no primeiro ano de curso, a ingestão de metilfenidato demonstrou-se superior aos outros psicoativos, sendo representada por $22 \%(n=57)$ do total, seguido pelo uso de energético com $17 \%(n=45)$. Verificando o uso no segundo ano de curso observou-se que tanto a ritalina $(n=17)$ quanto o energético $(n=$ 15) corresponderam a $6 \%$ do total, seguidos pelo uso de cafeína, ecstasy, maconha e outros tipos de psicoativos que tiveram $2 \%(n=4)$ de prevalência neste ano.

No terceiro ano de curso o uso de ritalina foi de $4 \%(n=10)$, seguido pelo uso de cafeína e energético com $3 \%(n=8)$ do total e a ingesta de ecstasy, maconha e outros psicoativos foi de $1 \%(n=3)$. A partir da análise do uso no quarto ano foi possível inferir que apenas o uso de cafeína foi de $1 \%(n=2)$, entretanto não houve ingestão de ecstasy, ritalina, energético, maconha e outros tipos de psicoativos. Indo ao encontro de tais dados o quinto e sexto ano também apresentaram porcentagens semelhantes, sendo incluído a cafeína na não ingestão (Tabela 3). 
Tabela 3 - Variáveis associadas ao uso de psicoestimulantes entre os alunos de medicina.

\begin{tabular}{ccccccc}
\hline $\begin{array}{c}\text { Ano de } \\
\text { curso }\end{array}$ & $\begin{array}{c}\text { Cafeína ou } \\
\text { Guaranina }\end{array}$ & Ecstasy & Metilfenidato & $\begin{array}{c}\text { Bebidas } \\
\text { energéticas }\end{array}$ & Maconha & Outros \\
\hline $\begin{array}{c}\text { Antes da } \\
\text { Graduação }\end{array}$ & $72 \%$ & $3 \%$ & $14 \%$ & $47 \%$ & $14 \%$ & $5 \%$ \\
\hline $1^{0}$ & $13 \%$ & $3 \%$ & $22 \%$ & $17 \%$ & $6 \%$ & $5 \%$ \\
\hline $2^{0}$ & $2 \%$ & $2 \%$ & $6 \%$ & $6 \%$ & $2 \%$ & $2 \%$ \\
\hline $3^{0}$ & $3 \%$ & $1 \%$ & $4 \%$ & $3 \%$ & $1 \%$ & $1 \%$ \\
\hline $4^{0}$ & $1 \%$ & $0 \%$ & $0 \%$ & $0 \%$ & $0 \%$ & $0 \%$ \\
\hline $5^{0}$ & $0 \%$ & $0 \%$ & $0 \%$ & $0 \%$ & $0 \%$ & $0 \%$ \\
\hline $6^{0}$ & $0 \%$ & $0 \%$ & $0 \%$ & $0 \%$ & $0 \%$ & $0 \%$ \\
\hline
\end{tabular}

Fonte: Zandoná I, et al., 2020.

Os principais motivos citados para o consumo de psicoestimulantes foram, devido a rotina $57,7 \%(n=153)$, aumento da capacidade cognitiva $24,1 \%(n=64)$, compensar a privação do sono $21,8 \%(n=58)$, diminuir o estresse $11,6 \%(n=31)$, sob prescrição médica $11,3 \%(n=30)$, curiosidade $6,7 \%(n=18)$, aumento da sensação de prazer 4,9\% $(n=13)$, e desinibição social 2,6\% $(n=7)$. Os dados da Tabela 4 denotam os efeitos percebidos com $\mathrm{o}$ uso dos estimulantes. Indicam um resultado positivo ao avaliar o aumento da concentração dos estudantes, sendo que $64,8 \%(n=248)$ relataram alguma melhora. Ao analisar a capacidade de memorização os resultados não foram tão satisfatórios, uma vez que $63,5 \%(n=244)$ relataram obter o efeito contrário ou não notar diferença.

Tabela 4 - Efeitos percebidos por alunos.

\begin{tabular}{|c|c|c|c|}
\hline Efeito & $\begin{array}{c}\text { Obteve o contrário } \\
(\%)\end{array}$ & $\begin{array}{c}\text { Melhorou } \\
\text { pouco/razoável } \\
(\%) \\
\end{array}$ & Melhorou muito (\%) \\
\hline $\begin{array}{l}\text { Aumento da } \\
\text { concentração } \\
(n=248)\end{array}$ & 5,2 & 47,9 & 16,9 \\
\hline $\begin{array}{l}\text { Capacidade de } \\
\text { memorização } \\
(n=244)\end{array}$ & 4,5 & 2,9 & 7,3 \\
\hline $\begin{array}{l}\text { Raciocínio } \\
\text { mais rápido } \\
(n=245)\end{array}$ & 4,4 & 34,6 & 8,5 \\
\hline $\begin{array}{l}\text { Diminuição do } \\
\text { sonodiurno } \\
(n=248)\end{array}$ & 4,4 & 5 & 19,7 \\
\hline $\begin{array}{l}\text { Diminuição } \\
\text { do Estresse } \\
(n=243)\end{array}$ & 25,9 & 1,6 & 4,5 \\
\hline $\begin{array}{c}\text { Diminuição da } \\
\text { fadiga e cansaço } \\
\text { físico } \\
\text { e mental } \\
(n=245)\end{array}$ & 19,6 & 35,1 & 5,7 \\
\hline $\begin{array}{l}\text { Aumento da } \\
\text { disposição e } \\
\text { bemestar } \\
(n=247)\end{array}$ & 14,1 & 46,1 & 8 \\
\hline
\end{tabular}

Fonte: Zandoná I, et al., 2020. 
Quando questionado sobre adquirir um raciocínio mais rápido, 56,6\% $(n=245)$ relataram possuir um efeito contrário ou não notaram diferença. Os resultados foram satisfatórios ao avaliar a diminuição do sono diurno, mostrando assim, que 69,7\% ( $n=248)$ obtiveram melhora. Porém, ao analisar a diminuição do estresse, $79,4 \%(n=243)$ dos estudantes destacaram um efeito antagônico ou não notaram diferença. Sobre a diminuição da fadiga, cansaço físico e mental, $59,2 \%(n=245)$ relataram efeito inverso ou indiferença. Além disso, $54,1 \%(n=247)$ relataram uma melhora no aumento da disposição e bem-estar (Tabela 4).

\section{DISCUSSÃO}

O uso de psicoestimulante para melhora da rotina acadêmica, aumento da capacidade cognitiva e compensação da privação do sono foram indicados por $57 \%$ dos participantes. Conforme essa prevalência, Morgan HL (2017) também apresentou dados semelhantes, uma vez que 47,4\% relataram as mesmas motivações pelo consumo. Esse cenário demonstra que há um estímulo devido à repercussão positiva dos fenômenos buscado, o que explica a frequência do uso.

A partir da análise, verifica-se que $65,2 \%$ do total dos entrevistados afirmaram utilizar algum psicoestimulante, sendo esse valor superior ao demonstrado em outros estudos, como o de Silveira et al (2015), que apresentou 57\% no levantamento de dados indicando o consumo. No entanto, ao realizar o comparativo com outros cursos da área da saúde observa-se uma redução, tendo apenas $9,8 \%$ dos estudantes usado psicoestimulantes (CORDEIRO N, 2017).

Os alunos do primeiro ano de medicina, apresentaram a estimativa de $62,5 \%$ de consumo, semelhante ao do estudo feito por Morgan HL (2017), que apresentou dados 69,2\%, e a amostra feita por Pires et al (2018) com $66,6 \%$ de predomínio nos no período de ingresso do curso bem como durante os primeiros anos. Apesar disso, a prevalência nos outros anos é discordante, uma vez que os alunos do terceiro ano apresentou uma porcentagem de $88,2 \%$ de uso, quarto ano $82,3 \%$ e o segundo ano com $71,2 \%$. Quando comparado ao estudo feito por Silveira RR, et al. (2015) os graduandos do segundo ano apresentaram 64,10\% de ingestão e os do terceiro ano $41,02 \%$, fato que corrobora para a hipótese de que os alunos da pesquisa fazem maior uso durantes os primeiros anos da graduação.

Ao restringir o levantamento ao uso de cigarros, o presente estudo apontou que $11,3 \%$ dos alunos possuem o hábito de fumar. Comparando com outros alunos da área da saúde, observou-se uma redução do consumo pelos acadêmicos da pesquisa, já que o curso de odontologia apresentou $24,92 \%$ e o de enfermagem 32,3\% de alunos que já usaram (BECKERT N, et al., 2016, SILVA BP, et al., 2012).

Avaliando-se o tempo de sono e sua qualidade, os participantes apresentaram média de sono diária de 6,4 horas, bem como, $49,8 \%$ classificaram a qualidade do sono como ruim. Sendo possível constatar uma semelhança com a média de 6,13 horas diárias apresentada na pesquisa de Cardoso et al (2009), porém, observou-se uma divergência quanto a qualidade do sono, que apenas $22,8 \%$ dos residentes e estudantes afirmaram ter a qualidade ruim. Além disso, em um estudo realizado em uma unidade local de saúde do Alto Minho - Portugal, apresentou resultados superiores, sendo $52,4 \%(n=103)$ dos participantes relataram dormir mal.

Ademais, com relação ao uso de remédio para dormir, há uma prevalência de $18,4 \%(n=49)$ de resposta positiva para os alunos. Semelhante ao resultado de 15,5\% na Universidade Extremo Sul do Brasil (MORGAN HL, 2017). Contrariamente a esses resultados, houve uma prevalência apenas de $8,6 \%$ de uso entre os graduandos de medicina de uma universidade de Botucatu-SP, demonstrando uma redução do uso dessas medicações (CORRÊA CC, et al., 2017).

Ao analisar o consumo de ecstasy, nota-se que seu uso não foi de grande relevância, sendo apenas 3\%, principalmente no início da graduação. Em contrapartida, um estudo sobre o uso de drogas entre universitários brasileiros e norte-americanos mostrou um uso de 9,7\% de ecstasy em uma população geral de brasileiros e 13,3\% entre norte-americanos (ECKSCHMIDT F, et al., 2013). Outro estudo realizado em Curitiba-PR apresentou uma grande discrepância, pois de 405 entrevistas, $62 \%(n=251)$ relataram fazer o uso com frequência da substância (TSUDA CA, et al., 2015). 
Referente ao uso de cafeína e guaranina, $72 \%$ ( $n=191)$ iniciaram seu consumo antes da graduação, tendo diminuído para $13 \%$ ( $n=35)$ durante a graduação. Semelhante a isso, na pesquisa de Morgan HL, et al. (2017), $67,1 \%(n=51)$ dos estudantes relataram ter iniciado o consumo de cafeína antes de ingressar na faculdade. Além disso, em uma pesquisa feita no Canadá com um público alvo com idades de 12 a 24 anos, dos 2055 entrevistados, $73,8 \%$ relataram consumir bebida energética e $84,7 \%$ relataram consumir café (HAMMOND D, et al., 2018).

Quanto ao uso de metilfenidato, a prevalência durante a graduação foi de $6,4 \%(n=17)$, sendo que do quarto ao sexto ano não houve utilização e $14 \%(n=35)$ relatam ter iniciado antes da graduação. Corroborando com esses dados, Morgan HL, et al. (2017), apresenta que, na Universidade do Extremo Sul, o uso de metilfenidato antes da graduação foi de $20 \%(n=40)$ e no momento da pesquisa foi de $5,5 \%(n=$ 11). Entretanto, em outro estudo, $34,2 \%$ dos estudantes do quinto e sexto ano afirmaram uso de metilfenidato, sendo esse número discordante da amostra obtida no estudo atual (SILVEIRA RR, et al., 2014). Ao analisar o índice de outros cursos, Alberto MS, et al. (2017), apresentou que 18\% dos estudantes de biomedicina, farmácia e enfermagem relataram uso, diante disso, verifica-se que a ingestão desse psicoativo também é relevante em outros cursos da área da saúde.

Já o uso de maconha o estudo demonstrou uma superioridade antes da graduação com $14 \%$ dos entrevistados, ocorrendo uma redução gradual após o início da faculdade para $6 \%$ e no ano conseguinte para $3 \%$. Dados discordantes foram encontrados no estudo feito por Vicenzi T, et al. (2017) onde a maconha foi descrita como o segundo psicoativo mais usado, sendo representado por $31,4 \%$ do total de entrevistados do curso de odontologia, bem como no trabalho realizado por Texeira RF, et al. (2008) em que houve 13,2\% de uso de maconha, no entanto, sem especificar o exato período de medicina.

\section{CONCLUSÃO}

O presente estudo demonstra que o uso de psicoestimulantes se encontra constante na rotina de muitos acadêmicos de medicina. Sendo que, o metilfenidato no meio acadêmico sem acompanhamento e prescrição médica já era esperado, pois é sabido que os estudantes buscam por uma melhora no aprendizado, se privando de sono e fazendo uso de psicoestimulantes concomitante ao metilfenidato, principalmente nos períodos de provas. Porém, a repercussão foi acima do esperado.

\section{AGRADECIMENTOS E FINANCIAMENTO}

Ao conselho Nacional de Desenvolvimento Científico e Tecnológico - CNPq, pelo auxílio financeiro com uma bolsa de Iniciação Cientifica dentro do Programa PIBIC/CNPq, n 128503/2018-1. A Instituição de Ensino Superior Privada pela oportunidade de estudos e utilização de suas instalações.

\section{REFERÊNCIAS}

1. AFFONSO RS, et al. o uso indiscriminado do cloridrato de metilfenidato como estimulante por estudantes da área da saúde da Faculdade Anhanguera de Brasília (fab). Infarma ciências farmacêuticas. 2016; 28(3): 166-172.

2. ALBERTO MS, et al. Só de metilfenidato entre acadêmicos no interior de Rondônia. Revista da Universidade Vale do Rio Verde, Três Corações - MG. 2017; 15(1): 170-178.

3. ALMEIDA DVP, et al. Efeitos cardiovasculares da cafeína: revisão de literatura. Revista ciências em saúde, Itajubá. 2013; 3(2).

4. ANDRADE JBC, et al. Contexto de formação e sofrimento psíquico de estudantes de medicina. Revista Brasileira de Educação Médica. 2014; 38(2): 231-242.

5. BALLISTRERI MC, et al. O uso de bebidas energéticas entre estudantes de educação física. Revista Latino Americana Enfermagem, Rosário, Argentina. 2008; 16.

6. BECKER TN, et al. Características do uso de produtos derivados do tabaco entre universitários do curso de Odontologia em uma Universidade de Curitiba. Revista de Odontologia da Unesp, [s.I.]. 2016; 45(1): 7-14.

7. CARDOSO HC, et al. Avaliação da qualidade do sono em estudantes de Medicina. Rev. bras. educ. med., Rio de Janeiro. 2009; 33(3): 349-355. 
8. CORDEIRON N, et al. Consumo de estimulantes cerebrais em acadêmicos da área da saúde na cidade de Ponta Grossa-PR. Visão Acadêmica, Ponta Grossa. 2017; 18(2): 23-45.

9. CORRÊA CC, et al. Sleep quality in medical students: a comparison across the various phases of the medical course. Jornal Brasileiro de Pneumologia, [s.I.]. 2017; 43(4): 285-289.

10. DA GRAÇA CSG. Consumo de estimulantes cerebrais nos estudantes de Medicina da Universidade da Beira Interior. Covilhã; 2013. Mestrado [Dissertação] - Universidade da Beira Interior.

11. DÁZIO EMR, et al. Use of alcohol and other drugs among male university students and its meanings. Ver Esc Enferm USP. 2016; 50(5): 785-791.

12. ECKSCHMIDT F, et al. Comparação do uso de drogas entre universitários brasileiros, norte-americanos e jovens da população geral brasileira: subtítulo do artigo. Jornal Brasileiro de Psiquiatria, Rio de Janeiro. 2013; 62(3): $199-207$.

13. HAMMOND D, et al. Adverse effects of caffeinated energy drinks among youth and young adults in Canada: a Webbased survey. Joule Inc., Canadá. 2018; 6(1): 19-25.

14. LAGE DC, et al. Uso de metilfenidato pela população acadêmica: revisão de literatura. Brazilian Journal of Surgery and Clinical Research - BJSCR. 2015; 10(3): 31-39.

15. MACHADO S, et al. Estudantes de medicina e as drogas: evidências de um grave problema. Revista brasileira de educação médica. 2015; 39(1): 159-167.

16. MENDES SV, et al. Estudo sobre o uso de drogas estimulantes entre estudantes de medicina. Ciência atual, Rio de janeiro. 2015; 5(1): 02-12.

17. MORGAN HL, et al. Consumo de Estimulantes Cerebrais por Estudantes de Medicina de uma Universidade do Extremo Sul do Brasil: Prevalência, Motivação e Efeitos Percebidos. Revista brasileira de educação Médica. 2017; 41(1):102-109.

18. PIRES MS, et al. O uso de substâncias psicoestimulantes sem prescrição médica por estudantes universitários. Revista Científica Fagoc Saúde, Ubá. 2018; 3(2): 22-29.

19. RODRIGUES M, et al. Avaliação da qualidade do sono em cuidados de saúde primários. Rev Port Med Geral Fam, Lisboa. 2014; 30(1): 16-22.

20. SILVA BP, et al. Uso do tabaco entre estudantes de enfermagem de uma faculdade privada. SMAD, Rev. Eletrônica Saúde Mental Álcool Drog. (Ed. port.) Ribeirão Preto. 2012; 8(2): 64-70.

21. SILVEIRA RR, et al. Patterns of non-medical use of methylphenidate among 5th and 6th year students in a medical school in southern Brazil. Trends In Psychiatry And Psychotherapy, [s.I.]. 2014; 36(2): 101-106.

22. TEIXEIRA RF, et al. Uso de substâncias psicoativas entre estudantes de odontologia da Universidade Federal do Espírito Santo. Ciência \& Saúde Coletiva, Brasil. 2008; 15(3): 655-662.

23. TSUDA CA, et al. Avaliação do padrão de uso de estimulantes em uma faculdade de Curitiba-PR. Cadernos da Escola de Saúde, Curitiba. 2015; 1(13): 116-132.

24. VINCENZI T, et al. Emergence of cannabis as the second most commonly used psychoactive substance among students. Journal Of Human Growth And Development, [s.I.]. 2017; 27(2): 244-252.

25. WEBB JR, et al. Prevalence of stimulant use in a sample of US medical students. Ann clin psychiatry 2013; 25(1): 27. 